\title{
Distress of unemployment lead to cancer
}

\begin{abstract}
Cancer related death is caused by population aging and stress of unemployment. The absence of cancer in the Eskimos upon his arrival in the Arctic, but a subsequent increase in the incidence of the disease as closer contact with Western civilization is known. Molecular pathogenesis of inhibitory antagonism and disorder in neural phase coordination of reentry, based on a rejection-related distress of unemployment, may lead to the inflammation-induced carcinogenesis. Unemployment leading to cancer pandemic may cause the decay of Western Civilization. In this year China has achieved a several leading positions in science with new supercomputer and research at the field of cancer.
\end{abstract}

Keywords: rejection-related distress, lesion of reentry, inhibitory antagonism, genetic polymorphisms, inflammation-derived carcinogenesis, apoptosis-autophagy dichotomy, NF-kB-IL-6-stat3 cascade
Volume 3 Issue 4 - 2017

Robert Skopec

AXON Researcher, Slovakia

Correspondence: Robert Skopec, Dubnik, AXON Researcher, Slovakia, Email zxcbnvm7@gmail.com

Received: October 22, 2016 | Published: July 19, 2017
Abbreviations: DAMPs, damage associated molecular pattern molecules; RAGE, receptor for advanced glycation end products; HMG-B1, high mobility group-B1; TGF- $\beta$, transforming growth factor- $\beta$; TNF, tumor necrosis factor; IL-1, interleukin-1; CNS, central nervous system; $\mathrm{CD}$, causal density; WTAIs, winner takes all instabilities; dACC, dorsal anterior cingulate cortex; sTNF- $\alpha$, soluble receptor for tumor necrosis factor- $\alpha$; NF- $\kappa \mathrm{B}$, nuclear factor- $\kappa \mathrm{B}$

\section{Introduction}

Sensory experience results in neurotransmitter release at synapses within a neural circuit and in turn leads to membrane depolarization with calcium influx into individual neurons. Calcium influx in the postsynaptic neuron can alter cellular function by activation of new gene transcription. Also activates a converging signaling pathways, or transcription factors in nucleus, this in turn control the expression of a large number of neuronal activity-regulated genes. The signaling pathways mediate activity-dependent transcription during experiencedependent neural development and plasticity. Neuronal activity regulates by the signal transduction pathways the activity-dependent gene expression program. Neuronal activity-regulated genes in turn influence this activity-regulated transcriptional program controlling neuronal development. ${ }^{1}$ The activity-regulated gene expression program can alter the function of specific synapses formed onto a neuron.

\section{Materials and methods}

In our recent study we have widely employed neural, immune and genetic approach using a hypothesis-driven method. It is including also the pathway analysis approach, based on results of a more comprehensive set mediators, genes, neural regions, etc. involved in a specific functional role of stress and inflammation in cancer. Also population-based association studies we use as powerful tools for examining genes with role in common multifactorial diseases that have a strong environmental component. We were looking for the strategy of genetic association studies to discuss the role of genetic polymorphisms that modulate inflammation and risk of cancer. Our understanding of relationship between inflammation and cancer is growing and central role in these processes by our opinion may play a focus on correlative studies. Cancers often arise as the end stage of inflammation, in adults, but not in children. Inflammatory cells and soluble factors are present in all tumors. The signs of this latent inflammation including tissue remodeling, angiogenesis, and other wound healing-like features are morphologic cues of invasive cancer. Recent evidence shows that these processes play a fundamental role in cancer development and progression and may predict the clinical behavior of a cancer better than the characteristics of the neoplastic cells themselves. ${ }^{2}$

In preclinical studies antibodies promote cancer development by initiating local chronic inflammatory responses mediated by antibody and immuno-complex deposition (in some cases the direct inhibition of Th1 responses by B cells play also a role). Cancer is a disorder of cellular and tissue architecture and reentry driven by redox and damage-associated molecular pattern molecules (DAMPs). Stressed cells release into the tumor microenvironments DAMPs that interact with their receptors (DAMP-R) as the receptor for advanced glycation end products (RAGE) on surviving, stressed cells within the tumor microenvironment; they drive a disordered tumor microenvironment. The disordered microenvironment favors tumor cell resistance to therapy by limiting apoptosis, enhanced stromagenesis, angiogenesis, and suppression of the adaptive immune response. One of hypotheses is that high mobility group B1 molecule (HMGB1) as a DAMP released into the tumor microenvironment plays a central role in the growth of tumors by its recruitment and activation of innate immune cells, with the resulting chronic inflammatory milieu promoting stromagenesis, angiogenesis, and cell proliferation, enhancing tumor growth. The critical interface between tolerance and immunity is dictated by oxidation or reduction of HMGB1. First released HMGB1 is reduced and promotes immunity, with resolution of inflammation; it is oxidized and inactivated, when a transforming growth factor- $\beta$ (TGF- $\beta$ ) is activated. Treatments and targets for inflammation have come to the fore and deserve attention. Preclinical and correlative studies may provide rationale for targeting factors and cytokines having a clear impact on inflammation within cancer, such as HMGB1, the RAGE and IL-1 $\beta$. Targeting these factors may decrease the incidence of cancers developing in the setting of chronic inflammation. ${ }^{2}$ 


\section{The nervous system also modulates immune respon-} ses

Deficiencies and excesses of the inflammatory response can cause morbidity and shorten lifespan: they can lead to infection and cancer. The inflammatory response may be more dangerous than the original inciting stimulus. Inflammatory stimuli activate sensory pathways that relay to the hypothalamus. Systemic increase in tumor-necrosis factor (TNF) mediates tissue injury by depressing cardiac output, inducing microvascular thrombosis and mediating systemic capillary leakage syndrome. TNF amplifies and prolongs the inflammatory response by activating other cells to release both cytokines: interleukin-1 (IL1), high mobility group B1 and mediators: reactive oxygen species, eicosanoids, nitric oxide which promote further inflammation and tissue injury. TNF is important for the complete expression of inflammation during invasion. Inflammatory stimuli can activate anti-inflammatory signals from the central nervous system (CNS). Inflammation in peripheral tissues alters neuronal signaling in the hypothalamus. ${ }^{3}$ There are evidences of a common molecular basis for communication, with cells from each system expressing signaling ligands and receptors from the other.

\section{Results}

Synapses between neuronal units with strongly correlated firing phase are then potentiated and synapses between neuronal units with weakly correlated phases are inhibited. With reentrant connections intact, distributions for all neurons are peaked at the same phase. With reentrant lesioned networks (triggered by the distress of unemployment as a consequence of social rejection) the probability distributions remain flat due to their phase independent input. Phase correlations between neural units are higher with intact reentrant connections than in lesion group. Lesions can be find by a Granger causality analysis, the causal density (cd) of a system can be calculated

as $c d=\alpha / n(n-1)$, where $\alpha$ is the total number of significant causal interaction and $n(n-1)$ is the total number of directed edges in a fully connected network with $n$ nodes, excluding self-connections. ${ }^{4,5}$

\section{Stress, inflammation and cancer: diseases of civiliza- tion}

Onset of malignancy may follow after emotional stress (disease of adaptation to civilization). Disasters of life, much trouble play role in the causation of cancer (the uneasy passions of the mind). Emotional factors are more common in sensitive and frustrated from competition leading to unemployment, lead to mental misery, sudden reverses of fortune, and represent a powerful cause of the cancer. The Bernoulli diminishing return intuition says that there are neural representations transforming their input (objective value) under a logarithmic type

of nonlinearity. A logarithmic function $u(x)=\log _{10}(x)$ is used as the expected utility. Paying a large amount of capacity in a high probability of making a loss and a small probability for a high win. ${ }^{6}$ The output is subject to additional independent noise of constant variance $c^{2}$. It can result to reversal effects with higher slope for losses than for gains. The variance in firing rate of neurons is approximately proportional to the mean firing rate. It was proposed a typical relationship between the mean and the variance of the inputs as $c_{i}^{2} \approx 1.5 I_{I_{y}}$. Influence of psychosocial environmental factors: emotional stress and cancer, envariance, stressful emotions can exert malignant growth. Unusual amount of self-dislike and distress (frequent feelings of hopelessness and helplessness) are also precursors to cancer. Subconsciously repeated same "fight or flight" responses are no longer appropriate and purposeful, adaptation lead to opportunism, automatic responses to stress don't seem to make any sense, form basis for diseases of civilization, (do not serve any useful or rational adaptive purpose). . $^{27-11}$ Development of malignancy is a more dramatic example of opportunism in the evolutionary process. Cancer was rare in antiquity, and it underscores the role of carcinogenic environmental factors in modern societies. ${ }^{12}$

\section{Emotional distress associated with an anticipated traumatic event}

Emotional distress associated with an anticipated traumatic event is often greater than result of the physical event itself (self-fulfilling prophecy). Emotional loss from the distress of unemployment is perceived as even greater stress than a physical preparation. What may result instead in a new growth in the form of neoplasia, which is malignant and beyond control. Neural regions processing rejectionrelated information are involved in inflammatory responding because help mount preparatory responses to potential physical injury. This is based on overlapping neural circuitry of physical and social pain. ${ }^{14}$ The ability to regenerate lost or injured tissue involves something more than a simple local response: repairing the lesion at the level of neural coordination in reentry! Statistics in loss of selected emotional relationships with death of a spouse and divorce providing evidence that stress can cause cancer. Depressed immune system is predisposing to cancer and explains some stress-related malignancies. It is not a new experience that cancer like insanity increases in a ratio to the civilization of the country, civilization is enhancing tendency to cancer. Asians immigrated to the West later in their life had lower risk of prostate cancer than compared with the White. Descending the phylogenetic scale, the incidence of cancer progressively increasing and is absent in primitive forms of life. In increase of cancer incidence predominates a competition based on winner-takes-all-instabilities (WTAIs), leading to the decay of Civilization.

To derive an equation for the dynamics of the winner-take-all instability, we express the dynamical variables as $x=x_{S S}+x_{c r} Y(T)+\ldots$ where $Y$ represents the slow dynamics along the critical eigenvector and $T$ is a slow time scale. The reflection symmetry of the system implies the dynamics of $Y$ should be invariant under the transformation $Y \rightarrow-Y$ and this switches the identity of $x_{1}$ and $x_{2}$ . The increase in input $I$ is common to both $x_{1}$ and $x_{2}$ leads to the developing decision in the winner-take-all system and is thus the bifurcation parameter. The linear growth rate of the spontaneous state must be proportional to the difference between the presynaptic input and the value of the input at the bifurcation with an unknown

prefactor, i.e. $\mu\left(I-I_{c r}\right)$. The difference in inputs $I_{1}-I_{2}$ breaks the reflection symmetry thereby introducing a constant term which, to first approximation, must be proportional to that difference with an unknown prefactor, i.e. $\eta\left(I_{1}-I_{2}\right)$. These two facts, coupled with the reflection symmetry, lead to the form of the equation describing the time evolution of $Y: \delta_{T} Y=\eta\left(I_{1}-I_{2}\right)+\mu\left(I-I_{c r}\right) Y+\gamma Y^{3}$, where $I=I_{c r}$ only when $\alpha=\beta$ identically, i.e. at point of genome instability, and $\delta_{T}$ is a time derivative with respect to the slow time $T$. For 
$I_{1}-I_{2}$ the equation is invariant under $Y \rightarrow-Y$ as it should be, $Y^{3}$ is the lowest order nonlinearity which obeys reflection symmetry. For more complex systems, which exhibit winner-take-all behavior, above equation captures the qualitative dynamics of the system near the bifurcation in general? In landmark experiments inhibition of apoptosis and autophagy in renal epithelial cells leads to increased necrotic cell death, genomic instability, inflammation, and rapid development of cancer. We are threatened with death from cancer for our inability to adapt to actual civilized living conditions, neural lesion in reentry triggered by the distress of unemployment, have implications for an increase in some malignancies as of psychosocial carcinogenesis. Stress-induced inflammation is implicated in serious disorders including depression and cancer, etc. Now we are beginning to know also the neurocognitive pathways in inflammatory responses to stress. Psychological stress has been underestimated for long period of time as possible causal factor in development of cancer. Animal and human research has shown that especially social stressors are very strong triggers of inflammation.

\section{Stress with threat of social rejection up-regulate in- flammatory activity}

Stress with threat of social rejection up-regulate inflammatory activity. Neural regions involved in processing rejection-related distress may relate to individual's magnitude of inflammatory responses to social stress. These brain regions include the dorsal anterior cingulate cortex (dACC) and the anterior insula. Greater activity in $\mathrm{dACC}$ has been associated with greater self-reported feelings of social distress. Neural regions associated with social rejection-related distress play role in inflammatory responses to stressors involving elements of social-evaluative threat and rejection. Greater activity in the dACC and bilateral anterior insula during social exclusion was associated with greater soluble receptor for tumor necrosis factor $\alpha$ (sTNF $\alpha$ RII) responses to the laboratorybased stressor. Greater activity in the right anterior insula was related to increase in interleukin-6 (IL-6). Inflammatory cytokines are released in response to risk of assault because can accelerate wound healing, reduce risk of infection, limit transmission of pathogens to others, and reduce risk for additional conflict. Neural encoding of peripheral inflammation gives to the para-limbic structures the ability to modulate inflammatory activity. Both, the ACC and the anterior insula have extensive efferent connections to the hypothalamus, enabling to influence inflammatory activity via endocrine pathways. These regions project also to brainstem autonomic control nuclei, by which peripheral inflammatory processes can be regulated by sympathetic and parasympathetic activity. Individual differences in magnitude of inflammatory responses to distress of unemployment help to explain the variability observed in susceptibility to disorders with an inflammatory component in certain_types of cancer and depression. Growing number of studies of emotional stimuli showing that the dACC and anterior insula are primary sites Slavich et al. ${ }^{13}$ of stress-related inhibitory antagonism Gold et al. ${ }^{14}$ activation.

\section{Molecular pathogenesis of inhibitory antagonism}

The environment is conductive to the growth of other bacteria within the gastric milieu, leading to sustained inflammation and oxidative/genotoxic stress. The cancer represents a classic example of inflammation-induced malignancy. Nuclear factor- $\kappa \mathrm{B}(\mathrm{NF}-\kappa \mathrm{B})$ and Stat3 proteins are transcriptional factors, which integrate stress signals and orchestrate immune responses also linked to carcinogenesis. NF- $\kappa$ B and Stat 3 control the expression of anti-apoptotic, proproliferative and immune response genes. These genes partly overlap and show transcriptional cooperation and inhibition between the two factors. Activation and interaction between NF- $\kappa$ B and Stat3 plays a key role in control of the dialog between the malignant cell and its microenvironment, with inflammatory/immune cells that infiltrate tumors. Cytokines induced in response to NF- $\kappa$ B in immune cells of the tumor microenvironment lead to Stat3 activation in both malignant and immune cells. Within malignant and pre-malignant cells Stat3 activates oncogenic functions; within inflammatory cells it may also suppress tumor promotion through its anti-inflammatory effects. Crosstalk between NF- $\kappa$ B and Stat 3 include cooperation of these factors at gene promoters/enhancers. NF- $\kappa \mathrm{B}$ dependent expression inhibitors of Stat3 activation and participation of Stat3 in inflammatory cells the negative regulation of NF- $\kappa$ B. Despite these variable and antagonistic interactions, NF- $\kappa$ B and Stat 3 cooperate to promote the development and progression of colon, gastric, and liver cancers. The proliferative and survival effects of IL- 6 are mediated by the transcription factor Stat3. The NF- $\kappa$ B-IL-6-Stat3 cascade is an important regulator of inhibitory antagonism.

Neurons in the CNS can synthesize and express TNF and IL1. These cytokines may participate on neuronal communication, which is bi-directional. Cytokines can activate hypothalamicpituitary release of glucocorticoids and, in turn, glucocorticoids suppress further cytokine synthesis. Cells of the immune system can produce neuropeptides (endorphins), acetylcholine and other neurotransmitters. Cytokines and glucocorticoids are also part of the inhibitory antagonism's cascade leading from stress to inflammation and cancer. The values of $y_{1}$ and $y_{2}$ are transformed through a nonlinear activation function $f(y)$ before they inhibit each other:

$d y_{i}=\left[-k y_{i}-w \sum_{j=I}^{N} f\left(y_{j}\right)+I_{i}\right] d t+c_{i} d W_{i} \quad$ integration starts from $y(0)=0$, an input unit with mean activity $I_{i}$, and independent white noise fluctuations $d W_{i}$ of amplitude $c_{i}$. The next part of the inhibitory antagonism's cascade, when these units also inhibit each other with a connection weight $w, k$ denotes the decay rate of the accumulated activity (leak), $N$ means the number of alternatives. Performance and dynamics of choice models suggests that in some cases the balance of inhibition and decay is not optimizing the performance, but rather it may be more profitable increase the inhibition parameter $w$, which increase inhibition of accumulators $y_{3}, y_{4}, y_{5}$ and thus prevent from the competition with $y_{1}$ and $y_{2}$ (for $N=5$ alternatives). ${ }^{6}$ This type of preemptive discrimination in favor of 'pointer states', which is suppressing further competitive behavior, is known from the Quantum

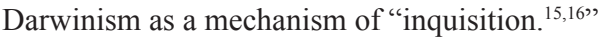

\section{Genetic polymorphisms directly influence the magni- tude of cytokine response}

Genetic polymorphisms directly influence inter individual variation in the magnitude of cytokine response and contribute to an individual's clinical outcome. Proinflammatory genotypes like $I L-1$ gene cluster polymorphism ( $I L-1 B$ encoding $I L-1 \beta$ ) enhances the risk of cancer progressively so that by time $3-4$ of the 
polymorphisms present, the ratio of gastric cancer increases 27 -fold. ${ }^{2}$ $H$. pylori are a prerequisite for the association of the polymorphisms with malignancy shows that inflammation is indeed driving carcinogenesis. Another part of the inhibitory antagonism mechanism is a paradoxical apoptosis-autophagy dichotomy in tumorigenesis and tumor progression. Autophagy contributes to "programmed cell survival" balancing and counter-regulating apoptosis. Alike the polycomb and trithorax group proteins having opposing effects on chromatin, and either inhibit or respectively activate gene expression in tumor biology. ${ }^{17}$ Neural regulation of inflammation depend from cholinergic inhibition of TNF, evidence indicates that these neural anti-inflammatory mechanisms also inhibit the release of IL-1, IL-18 and HMGB $1 .^{3}$ Loss of endogenous anti-inflammatory mechanisms converts normally protective, self-limited inflammatory response into an excessive, potentially deleterious response.

\section{Envariance of the microenvironment at atomic level}

The state of composite object (consisting of the system $S$ and the environment $E$ ) can be ignorant of the state of $S$ alone. Environment-assisted invariance, or envariance (at atomic level) based on symmetry allows observer to use perfect knowledge of $S E$ as a proof of his ignorance of $S:$ when a $u_{S}$ acting on $S$ alone, can be undone by a transformation acting solely on $E$, and the joint state of $S E$ is unchanged. This state is said "envariant" with respect to $u_{S}$ . Envariant properties not belong $S$ alone. Entanglement between $S$ and $E$ enables envariant and implies ignorance about $S$. Envariance is associated with phases of the Schmidt decomposition of the state representing $S E$. It anticipates the consequences of environment induced superselection ("einselection") of the preferred set of pointer states; they remain unperturbed to immersion of the system in the environment. The state of combined $S E$ expressed in the Schmidt form is: $|\psi S E\rangle=\sum_{k=1}^{N} \alpha_{k}\left|\delta_{k}\right\rangle\left|E_{k}\right\rangle$. Schmidt states are in an intimate relationship with the pointer states and have been regarded as "instantaneous pointer states. ${ }^{18 "}$ Quantum Darwinism brings new focus on the environment as a communication channel. This explains the emergence of objectivity. Even hazy environment will communicate a very clear image. ${ }^{16,19}$ The CNS receives sensory input from the immune system through both humoral and neural routes. The immune system detects microbial invasion and produces molecules that relay information to brain. TNF and other immunological mediators can gain access to brain centers devoid in the circumventricular region.

\section{Somatic sensory input into the CNS is organized so- matotopically}

Somatic sensory input into the CNS is organized somatotopically (sensory input from a discrete peripheral site is localized precisely in the ascending fibre pathway and brain). The first synapse for afferent vagus signals is in the nucleus tractus solitarius, and lesioning of this region impairs the development of IL-1-induced fever. The role for value-dependent synaptic activity differs from the valueindependent rule in that additional term, based on the activity and phase of the value system, modulates the synaptic strength changes. The synaptic change for value-dependent synaptic plasticity is:

$$
\Delta_{c_{i j}}(t+1)=\eta_{s_{i}}(t)_{s_{j}}(t) B C M(\Delta p) V(t) B C M_{i j}\left(\Delta_{i j}\right) \text { where } V(t) \text { is the }
$$

mean activity level in the value area $S$ at the time $t . B C M_{i j}$ function is different from the $B C M$ function above in that it uses the phase difference between area $S$ and the postsynaptic neuronal unit as input, $\Delta_{i j}(t)=\frac{\cos \left((2 \pi / n)\left(p_{i j}(t)-p_{i}(t)\right)+1\right.}{2}$, where $p_{i j}(t)$ is the mean phase in area $S$. When the both $B C M$ and $B C M_{i j}$ return to negative number, $B C M_{i j}$ is set to 1 to ensure the synaptic connection is not potentiated when both the presynaptic neuronal unit and value system are out of phase with the postsynaptic neuronal unit. Direct stimulation of the efferent vagus nerve inhibits the synthesis of TNF in spleen and attenuates serum concentration of TNF during endotoxemia. ${ }^{3}$ Inflammation-derived sensory input can be processed differentially in the brain, depending on the location of the inflammatory site and the character of the sensory signal. Vagus nerve activity can be relayed to medullary reticular formation, to the locus ceruleus and hypothalamus, leading to increased release of ACTH from anterior pituitary.

\section{Conclusion}

Manifestation of the inflammatory microenvironment is suppression of anti-tumor immune responses. Chronic inflammation promotes tumor development and is not the one response but instead represents a dynamic, continuously changing microenvironmental process with various effects at subsequent stages of tumorigenesis. Multiple factors in both the host and the malignant cells, the malignancy has impact on the inflammatory response and vice versa. Understanding these factors, and their relationship to treatment response, is including also a study of the unemployment distress. If we continue to close eyes over the role of unemployment in cancer pandemic, it will lead to decay of Western Civilization in relatively short time. In this year 2010 China has achieved a global leading position in science with new supercomputer and research at the field of cancer. ${ }^{20}$ We hypothesize that this puzzling increase in the incidence of cancer may be related to stress of civilization. Molecular pathogenesis of inhibitory antagonism and lesion in reentry (neural phase coordination) based on a rejection-related distress during competition may lead to the inflammation-derived carcinogenesis. Mechanism is triggered by interactive behavior of an appraisal of unit $P$ probabilities trade-off with environment. In a job-interview with 100 subjects, 1 of them will be the winner, and the 99 losers may get rejection-induced inflammations (precursor of cancer). Hypertrophy of one competition model: the winner-take-all type of competition is becoming less adaptive in $21^{\text {st }}$ century because lead to serious diseases including stress, inflammation and cancer.

\section{Acknowledgements}

None.

\section{Conflict of interest}

The author declares no conflict of interest.

\section{References}

1. Flavell SW, Greenberg ME. Signaling mechanisms linking neuronal activity to gene expression and plasticity of the nervous System. Annu Rev Neurosci. 2008;31:563-590

2. Demaria S, Pikarsky E, Karin M, et al. Cancer and inflammation: promise for biologic therapy. J Immunother. 2010;33(4):335-351. 
3. Tracey KJ. The inflammatory reflex. Nature. 2002;420(6917):853-859.

4. Seth AK, McKinstry JL, Edelman GM, et al. Visual Binding through Reentrant Connectivity and Dynamic Synchronization in a Brain-based Device. Cerebral Cortex, USA: Oxford University Press; 2004;14:11851199.

5. Seth AK, Izhikevich E, Reeke GN, et al. Theories and measures of consciousness: An extended framework. Proc Natl Acad Sci USA. 2006;103(28):10799-10804.

6. Bogacz R, Usher M, Zhang J, et al. Extending a biologically inspired model of choice: multialternatives, nonlinearity and value-based multidimensional choice. Philos Trans $R$ Soc Lond B Biol Sci. 2007;362(1485):1655-1670.

7. McCredie M, Williams S, Coates M. Cancer mortality in migrants from the British isles and continental Europe to new south Wales, Australia, 1975-1995. Int J Cancer. 1999;83(2):179-185.

8. World Cancer Report. In: Stewart BW, et al. editors. Lyo, France: IARC Publications; 2003.

9. Parkin DM, Bray F, Ferlay J, et al. Global cancer statistics. CA Cancer J Clin. 2005;55(2):74-108.

10. Elizabeth TH Fontham. Cancer Epidemiology and Prevention. 3rd ed In: Schottenfeld D, et al. editors. New York, USA: Oxford University Press; 2006. 469 p.
11. Chen LL, Blumm N, Christakis NA, et al. Cancer metastasis network and the prediction of progression patterns. Br J Cancer. 2009;101(5):749758

12. David AR, Zimmerman MR. Cancer: an old disease, a new disease or something in between? Nat Rev Cancer. 2010;10(10):728-733.

13. Slavich GM, Way BM, Eisenberger NI, et al. Neural sensitivity to social rejection is associated with inflammatory responses to social stress. Proc Natl Acad Sci U S A. 2010;107(33):14817-14822.

14. Gold JI, Shadlen MN. The neural basis of decision making. Annu Rev Neurosci. 2007;30:535-574.

15. Kelsey HT. Quantum Darwinism. The reality of reality? Los Alamos National Laboratory Newsletter. 2005;6(14):1-4.

16. Zurek WH. Quantum Darwinism. Nature Physics. 2009;5:181-188.

17. Mills AA. Throwing the cancer switch: reciprocal roles of polycomb and trithorax proteins. Nat Rev Cancer. 2010;10(10):669-682.

18. Zurek WH. arXiv: 0211.037 v1 [quant-ph]. India; 2002.

19. Zwolak M, Quan HT, Zurek WH. Quantum Darwinism in a hazy environment. Phys Rev Lett. 2009; 103:110402.

20. Wu M, Pareja JCP, Xu T. Interaction between Ras(V12) and scribbled clones induces tumor growth and invasion. Nature. 2010;463(7280):545548 . 